\title{
Modification of polypropylene filter with metal oxide and reduced graphene oxide for water treatment
}

\begin{abstract}
A hydrothermal method for the synthesis of reduced graphene oxide/titanium dioxide filter (RGO/TiO2) and reduced graphene oxide/zinc oxide filter ( $\mathrm{RGO} / \mathrm{ZnO}$ ) by using polypropylene (PP) porous filter is reported. Field emission scanning electron microscopy illustrated that the nanoparticles were uniformly distributed on the reduced graphene oxide nanosheets. Flexural tests showed that the physical properties of the modified filters have greater strength than the original filter. Thermogravimetric analysis revealed that the thermal property of the modified filters is the same as that of the original filter. Under a halogen lamp, the modified filter exhibited excellent photocatalytic degradation of methylene blue. The RGO/TiO2 filter maintained its ability to degrade MB efficiently, even after five cycles of photocatalysis.
\end{abstract}

Keyword: Adsorption; Graphene; Photocatalysis; Titanium oxide; Water filter; Zinc oxide 